\title{
Modeling structural plasticity in dendrites with multiple spine types Steven Baer ${ }^{1,3}$, Sharon Crook ${ }^{1,2,3}$ and Michael McCamy*1
}

Address: ${ }^{1}$ Department of Mathematics and Statistics, Arizona State University, Tempe, Arizona 85287, USA, ${ }^{2}$ School of Life Sciences, Arizona State University, Tempe, Arizona 85287, USA and ${ }^{3}$ Center for Adaptive Neural Systems, Arizona State University, Tempe, Arizona 85287, USA

Email: Michael McCamy* - camy@mathpost.la.asu.edu

* Corresponding author

from Seventeenth Annual Computational Neuroscience Meeting: CNS*2008

Portland, OR, USA. 19-24 July 2008

Published: II July 2008

BMC Neuroscience 2008, 9(SuppI I):PI04 doi:I0.I I86/I47I-2202-9-SI-PI04

This abstract is available from: http://www.biomedcentral.com/I47I-2202/9/SI/PI04

(c) 2008 Baer et al; licensee BioMed Central Ltd.

\section{Introduction}

Dendritic spines can change in number and shape in response to various physiological, behavioral or pathological states. This activity-dependent structural plasticity exists over a vast range of time scales, from minutes to days or weeks $[1,2]$. Although spines may have a continuum of shapes, evidence suggests that spines may cluster in defined groups by their shapes [3-6]. Further evidence suggests that spine morphology correlates with distance from the cell body $[3,4]$; proximal to the soma, where the dendrite has the largest diameter, spines have short necks and appear stubby, whereas distally, where the dendrite is thinner, spines have thinner and longer stems. At intermediate distances, a variety of spine types are seen including the intermediate mushroom-shaped spines.

We formulate a stage-structured population model for activity-dependent spines of three types: stubby (type-I), mushroom (type-II), and thin (type-III). Each spine type is characterized electrically by a spine stem resistance and chemically by a parameter that controls the level of calcium accumulation in the spine head. Transitions between spine types are driven by intraspine calcium levels that depend on local electrical activity. A continuum formulation based on the cable equation [7] represents a dendritic branch and includes the stage transitions between stubby, mushroom, and thin dendritic spines. The model allows for a study of the interaction between the many activity-dependent (active or passive) spines and for an investigation of the impact of their individual and collective dynamics on the output properties of the dendrite.
Simulations are run for both passive and active spines to investigate how dendritic diameter and synaptic input frequency influence spine morphology along the dendrite. In the passive case, the model is reduced to a system of ordinary differential equations because evidence shows spatially uniform repetitive synaptic input to passive spines, within an input region, drive spine restructuring within that region, with little or no effect on dendritic output and structural change outside the input region. This is in stark contrast to excitable spines where synaptic activity can drive restructuring and dendritic output far from the input region. Finally, we discuss how this model can be extended to study the formation and loss of spines as well as how to handle different input frequencies.

\section{References}

I. Nimchinsky EA, Sabatini BL, Svoboda K: Structure and function of dendritic spines. Annual Reviews Physiology 2002, 64:3I3-353.

2. Matsuzaki M: Factors critical for the plasticity of dendritic spines and memory storage. Neuroscience Research 2007, 57:I-9.

3. Jones EG, Powell TPS: Morphological variations in the dendritic spines of the neocortex. Journal of Cell Science 1969, 5:509-529.

4. Peters A, Kaiserman-Abramof IR: The small pyramidal neuron of the rat cerebral cortex. American Journal of Anatomy 1970, 1 27:32I-355.

5. Lee KJ, Kim H, Kim TS, Park S-H, Rhyu IJ: Morphological analysis of spine shapes of Purkinje cell dendrites in the rat cerebellum using high-voltage electron microscopy. Neuroscience Letters 2004, 359:2।-24.

6. Tyler WJ, Pozzo-Miller L: Miniature synaptic transmission and BDNF modulate dendritic spine growth and form in rat CAI neurones. Journal of Physiology 2003, 553.2:497-509.

7. Baer SM, Rinzel J: Propagation of dendritic spikes mediated by excitable spines: a continuum theory. Journal of Neurophysiology 1991, 65:874-890. 